\title{
Opinión de los docentes sobre el tratamiento de las competencias en los libros de texto de Ciencias de la Naturaleza en Educación Primaria
}

\author{
Javier Rodríguez Moreno ${ }^{1, a}$, Antonio de Pro Bueno ${ }^{2, b}, \mathbf{M}^{a}$ Dolores Molina Jaén ${ }^{3, c}$ \\ ${ }^{1}$ Departamento de Pedagogía, Universidad de Jaén, Jaén, España. \\ ${ }^{2}$ Departamento de Didáctica de las Ciencias Experimentales, Universidad de Murcia, Murcia, España. \\ ${ }^{3}$ Departamento de Psicopedagogía, Centro Universitario Sagrada Familia, Úbeda, España. \\ ajrmoreno@ujaen.es, ${ }^{b}$ nono@um.es, 'lomolin@hotmail.com
}

[Recibido: 13 septiembre2017. Revisado: 30 octubre 2017. Aceptado: 19 abril 2018]

\begin{abstract}
Resumen: Si hay una "seña de identidad" en los últimos programas oficiales, esta es la inclusión de las competencias. Sin embargo, la escasa concreción de cualquier reforma lleva al profesorado a acudir a los libros de texto para saber qué hacer en el aula. Ahora bien, ¿responden estos materiales de aprendizaje a lo que señala el currículum sobre la enseñanza de las competencias? Tras la última reforma educativa (LOMCE, 2013) y su posterior desarrollo curricular en la Comunidad Autónoma de Andalucía hemos querido conocer la opinión de algunos docentes sobre la adecuación al marco normativo y el nivel de logro competencial que presentan los libros de texto. Nos hemos ocupado de la Competencia en comunicación lingüística (CCL); la Competencia matemática y básicas en ciencia y tecnología (CMyCT) y Competencias sociales y cívicas (CSyC). Para ello, se elaboró un cuestionario y se pasó a unos maestros de Educación Primaria. Los resultados muestran las deficiencias existentes en el planteamiento actual que realizan los libros de texto y su distanciamiento de lo que marca el currículum oficial.
\end{abstract}

Palabras clave: Libros de texto; Ciencias de la Naturaleza; Currículum oficial; Educación Primaria; Competencias.

Teachers 'opinion on the treatment of competences in textbooks of natural sciences 2 in primary education

Abstract: If there is a "hallmark" in the latest official programs, this is the inclusion of the competencies. However, the scarce concreteness of any reform leads teachers to turn to textbooks to know what to do in the classroom. Now, do these learning materials respond to a model based on learning competencies? After the last educational reform (LOMCE, 2013) and its subsequent curricular development in the Autonomous Community of Andalusia we wanted to know the opinion of some teachers about the adequacy to the normative framework and the level of competence achievement of textbooks. We have dealt with the Competence in linguistic communication (CCL), Mathematical and basic competence in science and technology (CMyCT) and social and civic competences (CSyC). To do this, we developed a questionnaire and passed on to some Primary Education teachers. The results show the deficiencies existing in the current approach of textbooks and their distancing from what the official curriculum marks.

Keywords: Textbooks; Natural Sciences; Official Curriculum; Primary education; Competences.

Para citar este artículo: Rodríguez Moreno, J., Pro Bueno, A. y Molina Jaén, M.D. (2018). Opinión de los docentes sobre el tratamiento de las competencias en los libros de texto de Ciencias de la Naturaleza en Educación Primaria. Revista Eureka sobre Enseñanza y Divulgación de las Ciencias 18 (3), $3102 . \quad$ doi: 10.25267/Rev_Eureka_ensen_divulg_cienc.2018.v15.13.3102

\section{Situación de partida}

Las reformas educativas se suceden en España como productos gubernamentales. No es el fruto de un debate de especialistas ni una necesidad demandada por los profesionales ni una exigencia externa que nos obligue internacionalmente. Se quitan y se ponen en los documentos oficiales. Con la LOGSE, por lo menos se crearon los Centros de Profesores y, sin entrar en los resultados que se obtuvieron, se organizaron Planes de Formación que

\author{
Revista Eureka sobre Enseñanza y Divulgación de las Ciencias \\ Universidad de Cádi\%. APAC-Eureka. ISSN: 1697-011X \\ bttp://dx.doi.org/10.25267/Rev_Eureka_ensen_divulg_cienc.2018.v15.i3.3102 \\ http:/ / reuredc.uca.es
}


trataban de clarificar qué se pretendía, qué cambios había que introducir, cómo formar al profesorado, etc. Sin embargo, desde hace ya algún tiempo, los legisladores parecen pensar que es suficiente la publicación en el BOE o en el equivalente en las Comunidades Autónomas para la implantación automática en las aulas y los centros. Da la impresión de que hay dos currículos distintos: el que aparece en los documentos y el que acontece en la realidad.

En esta sucesión de reformas, contrarreformas y reformas de contrarreformas es difícil que los profesores lleguemos a saber qué tenemos que cambiar, qué debemos mantener y qué simplemente hemos de trasformar de lo que hacemos. Los vaivenes curriculares generan inestabilidad, perplejidad y probablemente cansancio. Y, en este contexto de desorientación colectiva, resulta lógico que los docentes recurran a un recurso que les informa de los contenidos, las actividades, etc. concretos para clarificar en qué consisten los programas oficiales: los libros de texto. Pero, sobre todo, da seguridad a los profesionales frente a la incertidumbre institucional.

La última reforma educativa (LOMCE, 2013) y los decretos y normativas que la desarrollan han recibido críticas desde amplios sectores de la sociedad y, por supuesto, del profesorado (Pro, 2013a; Trujillo, 2014; Bolivar, 2015). Se han incorporado términos a la "jerga pedagógica" como competencias básicas, estándar de aprendizaje, pruebas diagnósticas... Pero ¿ha cambiado realmente algo de lo que se hacía en las aulas?

\section{Fundamentos del trabajo}

Nuestro trabajo lo situamos en la Educación Primaria. Aunque la LOMCE considera que uno de los objetivos de este periodo educativo es la preparación de los estudiantes para la Educación Secundaria, creemos que es una etapa con unas finalidades propias. De hecho, aunque sus objetivos se han formulado de manera diferente en cada reforma, podríamos decir que pretende ser una herramienta social para incorporar al alumnado de 6-12 años a una sociedad democrática, multicultural, plural, tecnológica y cambiante.

Una característica común a ambas etapas podría ser el papel que juega el libro de texto en las mismas. Como ya se ha comentado en otros trabajos, este recurso ha sido algo más que un material de aprendizaje: se ha confundido con el currículum oficial, ha servido para clarificar cambios que se exigían y no se entendían, ha permitido actualizar científicamente al profesorado, ha dado seguridad a los docentes... (Pro 2013b; Pro y Rodríguez, 2014; Pro, Pro y Serrano, 2016). De ahí la importancia de indagar sobre los mismos.

\section{Los libros de texto en la enseñanza en la Educación Primaria}

El libro de texto está ligado al aprendizaje en el aula desde el siglo XV con el descubrimiento de la imprenta y desde luego se ha considerado determinante en el proceso de enseñanzaaprendizaje (Valera, 2010). Martínez Bonafé (2002) lo definió como el protagonista de un enfoque tecnológico de la enseñanza que hace tiempo se demostró estéril, que se basa en la trasmisión de la información y que conlleva un componente ideológico de lo que significa la educación obligatoria, pero, según este autor, en la sociedad actual, la construcción social de las subjetividades se realiza en otros contextos y por otros medios, por lo que no es eficaz dicha transmisión ideológica.

El análisis de los libros de texto se ha enfocado desde distintos puntos de vista: el estilo (Strube, 1989), el contenido (Martínez Bonafé, 2002; Cortés, 2006; Urones, Escobar y Vacas, 2013; Qadeer, 2014), las exigencias cognitivas (Shayer y Adey, 1984), las ilustraciones (Constable, Campbell \& Brown, 1988; Martínez y Gil, 2001; Perales, 2006; López y Postigo, 2014; 2016; Rodríguez, de las Heras, Romero y Cañal, 2014), las actividades (García y 
Martínez, 2003; Pro, Sánchez y Valcárcel, 2008), los valores que trasmiten (Rodríguez Lorenzo, 2015), los fundamentos didácticos en el área de Conocimiento del Medio (Travé, Estepa y Delval, 2015) o en Educación Infantil (Molina y Rodríguez, 2016) o desde la perspectiva de ser una herramienta en la actualización científica del profesorado (Molina, Rodríguez, Pérez y Callado, 2015; Martin, Jiménez y Prieto, 2015). En ellos se han identificado aspectos positivos y otros cuestionables sobre su utilidad en el aula de Ciencias.

Sin embargo, a pesar de las críticas recibidas, la Federación de Editores Españoles ANELE (2013) ha aportado datos interesantes y actualizados: considera al libro de texto la principal herramienta didáctica de los docentes, ya que el 81,3\% de ellos reconocen emplearlo bastante o mucho en su labor diaria. Además, las familias lo consideran imprescindible en la educación de sus hijos, tanto en los centros educativos como en el hogar $(71,9 \%)$, con lo que podemos afirmar que este recurso está plenamente asentado en el proceso de enseñanza al alumnado español.

En este trabajo, no cuestionamos la utilización mayoritaria del libro de texto en Educación Primaria y en concreto en el área de Ciencias de la Naturaleza. La presencia e importancia de este recurso hacen que su contenido no pueda ser ignorado en la puesta en práctica de cualquier cambio curricular. Pero, ¿cómo se han recogido en los libros de texto la "aparición" de las competencias y los estándares de aprendizaje? ¿qué han modificado las editoriales con los nuevos planteamientos curriculares?

\section{Las competencias en el área de ciencias de la naturaleza en Educación Primaria}

Ferreyra et al. (2008) manifestaban que la escuela enseña contenidos del siglo XIX, con profesionales del XX para alumnado del siglo XXI. Basándose en ello, defendían la necesidad de pasar de unos programas oficiales centrados en la acumulación y memorización de información a otros más acordes con unas necesidades diferentes a las de antaño.

Con las últimas reformas -la LOE y la LOMCE- ha irrumpido con fuerza la idea de que es preciso un currículum basado en la adquisición y el desarrollo de competencias (Villarini, 2000; Perrenoud, 2004; Ferreyra y Millén, 2006; Rodríguez, Ruiz y Guerra, 2007; Pro y Miralles, 2009; Pro y Rodríguez, 2011; Cano, 2015; Ferreira, 2015). Pero, como indican Monereo y Pozo (2007), formular competencias en términos generales, universales, es el mejor camino para que resulten estériles. Si queremos trasladarlas al trabajo de aula es preciso identificar las principales capacidades que se deben desarrollar, concretar cómo podemos hacerlo y, planificar intervenciones intencionadas que permitan que sean desarrolladas por los estudiantes.

No obstante, en la legislación actual, se ha optado por otro camino: introducir nuevos elementos curriculares; en concreto, los estándares de aprendizaje (los resultados de aprendizaje esperados de los estudiantes que todos deben alcanzar a lo largo de su trayectoria escolar) y los indicadores (herramientas para determinar el nivel de logro de un estándar). No sabemos cómo son interpretadas ambas por los docentes, pero formalmente son los elementos referenciales que han clarificado las competencias que deben trabajarse en las materias de carácter científico.

En nuestra Comunidad Autónoma, el currículum de Educación Primaria fue desarrollado por la Orden de 17 de marzo de 2015 (CEJA, 2015). En este documento, se contemplan los Objetivos (8) para el Área de Ciencias de la Naturaleza. Se establece el Marco de Desempeño en el que se relacionan dichos objetivos, los criterios de evaluación para cada ciclo (28 criterios) y por etapa (21 criterios), y los correspondientes estándares de aprendizaje (81), que nos parecen desproporcionados para sólo un área de conocimientos. Luego se plantea el 
Desarrollo Curricular del Área, diferenciando los tres ciclos, que son el referente de nuestro trabajo. A partir de los Criterios de evaluación (9 para el Primer ciclo, 10 para el Segundo y 9 para el Tercero), se plantean: orientaciones y justificaciones, los objetivos del área para la etapa con los que se relacionan, los contenidos por ciclo, las competencias y sus indicadores. Por último, en relación a los contenidos, podemos decir:

a) Primer ciclo. Parece que la base del conocimiento científico planteada por los legisladores está en el mundo real, los objetos, las imágenes, los fenómenos.... Esto justificaría que los criterios de evaluación de este periodo estén centrados en la observación, la identificación, la localización, el reconocimiento, la diferenciación, la clasificación... y la iniciación a la experimentación a través de montar y desmontar aparatos simples de la vida cotidiana. También tiene una presencia importante la creación de hábitos saludables, de respeto y conservación del medio, el desarrollo de la curiosidad... Además, un elemento que nos parece fundamental, presente en todos los ciclos, es la alusión al trabajo colaborativo, un eje importante en el aprendizaje de las ciencias que parece tradicionalmente olvidado en muchos libros de texto.

b) Segundo ciclo. Parece que el paso por los siguientes cursos académicos debe hacer que el alumnado refuerce la observación, la localización, la clasificación... y, además, que empiece a recoger y analizar datos, y a conocer algunas leyes que rigen fenómenos físicos o naturales. Este conocimiento debería permitir que se valore, desde las edades más tempranas, el progreso científico del ser humano gracias a la comprensión y utilización de sus logros; además, se mantienen los hábitos saludables, la higiene, la conservación del medio, la curiosidad... Nuevamente se habla de realizar proyectos colaborativos.

c) Tercer ciclo. Parece que, una vez que la capacidad de observación se ha asentado, deben trabajarse las interpretaciones y explicaciones de las mismas, las predicciones, el establecimiento de conjeturas, la búsqueda de información, la elaboración de informes... Además, se abren paso habilidades de investigación más complejas ya que, además de la realización de experiencias, se propone el diseño y la construcción de objetos y aparatos que pueden ser realizados de forma grupal o individual. Nos parece que refuerza la idea de un trabajo basado en la metodología científica desde los primeros años de la Educación Primaria, sin olvidar el consumo responsable, el trabajo cooperativo, la curiosidad y la valoración de los avances de la Ciencia.

Ahora bien, el quehacer didáctico o las estrategias metodológicas que realiza un docente no tiene las mismas características si se planifica la clase desde un trabajo por competencias (Méndez, Méndez y Fernández, 2015) que si se hace para seguir un libro de texto "clásico". Por ello, necesitamos conocer qué piensan los maestros sobre los libros de texto "actuales" que usan y sobre la contribución de estos a la adquisición de competencias que establece el currículum oficial.

\section{Diseño del trabajo}

Partiendo de las consideraciones de Mc Millan y Schumacher (2005) sobre las características de los problemas de una investigación, nos hemos planteado: ¿Responden los libros de texto a los indicadores de los criterios de evaluación y al trabajo por competencias que exige la normativa actual? ¿Qué piensan los profesores en ejercicio sobre este tema?

Para aportar respuestas a estos, hemos analizado las valoraciones que realizaban unos docentes sobre los libros de texto que utilizaban en sus clases. Correspondían a cuatro editoriales muy presentes en centros de Educación Primaria (por orden alfabético): Anaya, Edelvives, Santillana y SM. En cada editorial hemos diferenciado los manuales de cada ciclo. 
Ahora bien, son muchos los elementos sobre los que podemos estudiar cuál es la opinión de los docentes: los objetivos, los contenidos, los criterios de evaluación, los estándares... En este trabajo, nos hemos centrado en dos:

- la adecuación del contenido de los libros de texto a los indicadores de los criterios de evaluación del currículum.

- la adecuación a las competencias, implícitas en los indicadores de los criterios de evaluación, del currículum.

Se trata, por tanto, de un estudio de carácter descriptivo. No obstante, no pretendemos generalizar los resultados, lo que exigiría técnicas de muestreo de otra naturaleza.

\section{Participantes y contexto}

La investigación se realizó en 12 centros públicos de las provincias de Jaén (La Carolina, Linares, Vilches y Jaén capital) y Granada (Atarfe, Chauchina, Otura y Granada capital). Todos eran de "línea 2" menos uno de "línea 1". Una característica común era que tenían numerosos proyectos innovadores; de hecho, el profesorado parecía comprometido con su profesión, concienciado en la importancia de la formación continua y trabajaban con planteamientos compartidos y coordinados.

La experiencia se llevó a cabo durante el curso escolar 2015-16. El número total de docentes que participaron en esta investigación fue de 66, distribuidos de la siguiente manera: 20 del primer ciclo, 22 del segundo y 24 del tercero. La distribución por ciclo y editorial se recoge en la Tabla 1.

Tabla 1. Distribución de participantes por editorial y ciclo

\begin{tabular}{|c|c|c|c|c|}
\hline & EDIT1 & EDIT2 & EDIT3 & EDIT4 \\
\hline Primer Ciclo & 8 & 8 & 3 & 1 \\
\hline Segundo Ciclo & 8 & 8 & 5 & 1 \\
\hline Tercer Ciclo & 8 & 8 & 7 & 1 \\
\hline
\end{tabular}

Consideramos que se trata de una muestra incidental ya que, en la selección, se priorizó la predisposición positiva a participar en nuestro trabajo. Tratamos de que hubiera un número semejante de participantes por ciclo. En cuanto a las editoriales, la mayor presencia de dos (EDIT1 y EDIT2) respecto a las otras (EDIT3 y, sobre todo, EDIT4) no ha sido buscada por los investigadores.

Casi la totalidad llevaban varios años como funcionarios con destino definitivo, aunque también había algún interino. En cuanto a la edad y el género había variedad, los docentes tenían una media de 39.9 años $(\sigma=7.6)$ y sexo femenino (19 hombres y 47 mujeres).

\section{Instrumento de recogida de información}

Elaboramos un instrumento de recogida de información para que los docentes pudieran expresar sus opiniones sobre los libros de texto. A partir de los criterios de evaluación y de sus correspondientes indicadores que el currículum oficial contempla para la enseñanza de las Ciencias en la Educación Primaria, recogidos en la Orden de 17 de marzo de 2015.

Se plantearon tres (uno para cada ciclo). El primer ciclo tiene nueve criterios de evaluación con 27 indicadores, el segundo tiene diez criterios con 29 indicadores y el tercero tiene nueve criterios con 25 indicadores; en total, 81 indicadores. Se usaron escalas Likert en cada 
indicador y las opciones de respuesta oscilaron entre 1 y 4 (siendo 1, nada; 2, poco; 3, bastante; y 4, mucho).

En cuanto a la validez del contenido, se realizó mediante un juicio de expertos. Se solicitó a 5 maestros (con más de 10 años de experiencia) y 5 profesores de varias universidades (un Catedrático y 4 Profesores Titulares de Universidad del área de Didáctica y Organización Escolar) que llevasen a cabo una valoración del instrumento, estableciendo las consideraciones oportunas para mejorarlo. Una vez incorporadas las sugerencias -casi todas de formatoquedaron configurados los tres instrumentos; uno se recoge en el Anexo 1.

Luego, se procedió a la aplicación de una prueba piloto a 10 docentes de la provincia de Jaén. Contrastamos la variabilidad (mediante la varianza $\sigma^{2}$ ) entre los ítems y estudiamos la fiabilidad mediante el coeficiente Alfa de Cronbach (Tabla 2).

Tabla 2. Coeficiente Alfa de Cronbach

\begin{tabular}{|c|c|c|}
\hline $\begin{array}{c}\text { 1er. Ciclo de Educación } \\
\text { Primaria }\end{array}$ & $\begin{array}{c}2^{\circ} \text { Ciclo de Educación } \\
\text { Primaria }\end{array}$ & $\begin{array}{c}\text { 3er. Ciclo de Educación } \\
\text { Primaria }\end{array}$ \\
\hline Alfa de Cronbach $=0,879$ & Alfa de Cronbach $=0,920$ & Alfa de Cronbach $=0,843$ \\
\hline
\end{tabular}

En general, 0.80 se considera un valor aceptable (Gliem \& Gliem, 2003). Por ello, podemos considerar que los tres instrumentos se consideran estadísticamente fiables. Como hemos dicho, hay dos cuestiones en las que hemos centrado nuestro estudio:

a) respecto a la adecuación de los textos a los indicadores, la valoración era directa. A partir de la realizada por los docentes en cada uno, podemos señalar cuál es el grado de adecuación global al currículum oficial percibido por los participantes, por ciclos y por editoriales.

b) en cuanto a la adecuación a las competencias oficiales, el proceso era diferente. En primer lugar, hemos elegido aquellas con mayor presencia en el Área de Ciencias de la Naturaleza: la Competencia en comunicación lingüística (CCL); la Competencia matemática y básicas en ciencia y tecnología (CMyCT) y Competencias sociales y cívicas (CSyC). Luego, aunque podíamos utilizar diferentes estrategias nos hemos apoyado en el propio currículum oficial; en este documento, se establece una correspondencia entre cada indicador y las competencias a las que va asociado o a las que contribuye su adquisición.

Tras una explicación detallada oral de lo que debían realizar, los cuestionarios fueron cumplimentados por los docentes en formato impreso. Para ello, nos pasamos por los centros la tarde que tenían dedicada a reuniones, tutoría... para que nos los rellenaran. Tardamos aproximadamente un mes y medio en tener los datos de todos los docentes.

\section{Resultados}

Como decíamos, nuestro objetivo es conocer qué opinaban los docentes sobre la adecuación de los libros de texto a dos elementos: los indicadores y las tres competencias mencionadas.

\section{A) En relación con los indicadores}

En la Tabla 3, se recogen las opiniones sobre los indicadores del Primer, Segundo y Tercer ciclo. La nomenclatura de los indicadores (primera columna) es la del documento oficial. Dado que se trata de una escala Likert -ordinal- recogemos la Mediana y los cuartiles Q1 y Q3. Se han señalado los valores de la Mediana iguales o superiores a 2,25 (amarillo) e iguales e inferiores a 1,5 (verde); también los indicadores con mayor rango inter-cuartil (azul claro), lo que refleja una mayor heterogeneidad en las opiniones de los participantes. 
Tabla 3. Opiniones de los docentes en relación con los indicadores de los tres ciclos

\begin{tabular}{|c|c|c|c|c|c|c|c|c|c|c|c|}
\hline & Q1 & $\begin{array}{c}\text { Median } \\
\text { a }\end{array}$ & Q3 & & Q1 & Mediana & Q3 & & Q1 & Mediana & Q3 \\
\hline$\overline{C N 1.1 .1}$ & 1,25 & 2,00 & 3,00 & CN2.1.1 & 1,00 & 1,50 & 2,00 & CN3.1.1 & 1,00 & 2,00 & 2,00 \\
\hline CN1.1.2 & 1,00 & 2,00 & 2,00 & CN2.1.2 & 1,00 & 2,00 & 2,00 & CN3.1.2 & 1,00 & 2,00 & 2,00 \\
\hline CN1.1.3 & 1,25 & 2,00 & 2,00 & CN2.1.3 & 1,00 & 2,00 & 2,00 & CN3.2.1 & 1,00 & 2,00 & 2,00 \\
\hline CN1.2.1 & 1,25 & 2,00 & 2,75 & CN2.2.1 & 1,00 & 2,00 & 2,00 & CN3.2.2 & 1,00 & 2,00 & 2,00 \\
\hline CN1.2.2 & 1,00 & 2,00 & 2,00 & CN2.2.2 & 1,00 & 2,00 & 2,00 & CN3.2.3 & 1,00 & 2,00 & 2,00 \\
\hline CN1.2.3 & 1,00 & 2,00 & 2,75 & CN2.2.3 & 1,00 & 2,00 & 2,25 & CN3.2.4 & 1,00 & 2,00 & 2,00 \\
\hline CN1.2.4 & 1,00 & 2,00 & 2,75 & CN2.2.4 & 1,00 & 2,00 & 2,00 & CN3.2.5 & 1,25 & 2,00 & 2,00 \\
\hline CN1.3.1 & 1,00 & 2,00 & 2,00 & CN2.3.1 & 1,00 & 2,00 & 2,25 & CN3.3.1 & 1,00 & 2,00 & 2,00 \\
\hline CN1.3.2 & 2,00 & 2,00 & 2,00 & CN2.3.2 & 1,00 & 2,00 & 2,00 & CN3.3.2 & 1,00 & 2,00 & 2,00 \\
\hline CN1.4.1 & 2,00 & 2,50 & 3,00 & CN2.3.3 & 2,00 & 2,00 & 3,00 & CN3.3.3 & 1,00 & 2,00 & 2,00 \\
\hline CN1.4.2 & 2,00 & 2,00 & 2,75 & CN2.4.1 & 1,00 & 1,00 & 2,00 & CN3.4.1 & 1,25 & 2,00 & 2,75 \\
\hline CN1.5.1 & 2,00 & 2,00 & 3,00 & CN2.4.2 & 1,00 & 2,00 & 2,00 & CN3.4.2 & 1,00 & 2,00 & 2,00 \\
\hline CN1.5.2 & 1,00 & 2,00 & 3,00 & CN2.4.3 & 1,00 & 2,00 & 2,00 & CN3.4.3 & 2,00 & 2,00 & 2,00 \\
\hline CN1.5.3 & 2,00 & 2,00 & 3,00 & CN2.5. & 1,00 & 2,00 & 2,25 & CN3.5.1 & 1,00 & 2,00 & 2,00 \\
\hline CN1.6.1 & 2,00 & 2,00 & 3,00 & CN2.5.2 & 1,00 & 2,00 & 2,00 & CN3.5.2 & 1,00 & 2,00 & 2,00 \\
\hline CN1.6.2 & 2,00 & 2,00 & 3,00 & CN2.5.3 & 1,00 & 2,00 & 2,00 & $\mathrm{CN} 3.5 .3$ & 1,00 & 1,50 & 2,00 \\
\hline CN1.6.3 & 2,00 & 2,00 & 2,00 & CN2.5.4 & 1,00 & 2,00 & 2,00 & CN3.5.4 & 1,00 & 1,00 & 2,00 \\
\hline CN1.6.4 & 1,25 & 2,00 & 3,00 & CN2.6.1 & 1,00 & 2,00 & 2,00 & CN3.6.1 & 1,00 & 2,00 & 2,00 \\
\hline CN1.7.1 & 1,25 & 2,00 & 2,75 & CN2.6.2 & 1,00 & 2,00 & 2,00 & CN3.6.2 & 1,00 & 2,00 & 2,00 \\
\hline CN1.7.2 & 1,00 & 2,00 & 2,00 & CN2.6.3 & 1,00 & 2,00 & 2,00 & $\mathrm{CN} 3.7 .1$ & 1,00 & 2,00 & 2,00 \\
\hline CN1.7.3 & 1,25 & 2,00 & 2,00 & CN2.7.1 & 1,00 & 2,00 & 2,00 & $\mathrm{CN} 3.7 .2$ & 1,00 & 1,50 & 2,00 \\
\hline CN1.8.1 & 2,00 & 2,00 & 3,00 & CN2.7.2 & 1,00 & 2,00 & 2,00 & CN3.8.1 & 1,00 & 2,00 & 2,00 \\
\hline CN1.8.2 & 1,25 & 2,00 & 3,00 & CN2.8.1 & 1,00 & 2,00 & 2,00 & CN3.8.2 & 1,00 & 2,00 & 2,00 \\
\hline CN1.8.3 & 2,00 & 2,00 & 3,00 & CN2.8.2 & 1,75 & 2,00 & 2,00 & CN3.9.1 & 1,00 & 2,00 & 2,00 \\
\hline CN1.8.4 & 1,25 & 2,00 & 2,75 & CN2.8.3 & 2,00 & 2,00 & 2,00 & CN3.9.2 & 1,00 & 2,00 & 2,00 \\
\hline CN1.9.1 & 1,25 & 2,00 & 2,75 & CN2.9.1 & 1,00 & 2,00 & 2,00 & & & & \\
\hline CN1.9.2 & 2,00 & 2,00 & 2,00 & CN2.9.2 & 1,00 & 1,50 & 2,00 & & & & \\
\hline & & & & \begin{tabular}{|l} 
CN2.10. \\
1 \\
\end{tabular} & 1,00 & 2,00 & 2,25 & & & & \\
\hline & & & & \begin{tabular}{|l}
$\mathrm{CN} 2.10$. \\
2 \\
\end{tabular} & 1,75 & 2,00 & 3,00 & & & & \\
\hline & & & & & & & & & & & \\
\hline
\end{tabular}

Consideramos que un indicador tiene una presencia "muy alta" cuando la mediana es superior a 3,5; una presencia "alta" cuando oscila entre 2,76 y 3,5 (adelantamos que no encontramos ninguno de estas dos categorías); una presencia "media", cuando la mediana oscila entre 2,75 y 2,26; una presencia "baja" cuando oscila entre 2,25 y 1,51; y "muy baja" si es igual e inferior a 1,5. En cuanto a la variabilidad, destacaremos los indicadores con un rango inter-cuartil mayor de 1,25 .

A la vista de los valores obtenidos podemos decir que:

a) En 1er ciclo, solo un indicador de los libros analizados fue valorado con una "presencia media".

CN.1.4.1. Conoce y utiliza de forma adecuada diferentes instrumentos para la observación y el estudio de los seres vivos.

No hubo indicadores con "presencia muy baja". Sin embargo, hubo 10/27 indicadores con una heterogeneidad destacable. Algunos ejemplos fueron: 
CN.1.1.1. Obtiene información y realiza de manera guiada, pequeñas experiencias o experimentos, estableciendo conjeturas respecto de sucesos que ocurren de forma natural o respecto de los que ocurren cuando se provocan.

CN.1.2.4. Identifica emociones y sentimientos propios, de sus compañeros y de los adultos, manifestando conductas pacíficas.

CN.1.6.4. Realiza sencillas experiencias y elabora textos, presentaciones y comunicaciones como técnica para el registro de un plan de trabajo, comunicando de forma oral, escrita y audiovisual las conclusiones.

CN.1.8.4. Identifica los elementos básicos de un ordenador y se inicia de forma guiada en el uso de internet.

CN.1.9.1. Monta y desmonta algunos objetos y aparatos simples, describiendo su funcionamiento, piezas, secuencia de montaje y explicando su utilización de forma segura.

b) En $2^{\circ}$ ciclo, no hubo indicadores con presencia media o superior. Sin embargo, hubo 3/29 con una "presencia muy baja".

CN.2.4.1. Muestra conductas de comportamiento activo en la conservación, respeto y cuidado de los seres vivos y de su hábitat.

CN.2.1.1. Obtiene y contrasta información de diferentes fuentes, para plantear hipótesis sobre fenómenos naturales observados directa e indirectamente y comunica oralmente y por escrito de forma clara, limpia y ordenada, usando imágenes y soportes gráficos para exponer las conclusiones obtenidas.

CN.2.9.2. Planifica y construye alguna estructura que cumpla una función aplicando las operaciones matemáticas básicas en el cálculo previo, y las tecnológicas (dibujar, cortar, pega, etc.).

En este ciclo, disminuye la heterogeneidad; solo 5/29 tuvieron un rango inter-cuartil con heterogeneidad destacable. Algunos ejemplos fueron:

CN.2.2.3. Adopta actitudes para prevenir enfermedades y accidentes, relacionándolos con la práctica de bábitos saludables.

CN.2.10.1. Conoce y explica algunos de los grandes descubrimientos e inventos de la bumanidad y su influencia en el hogar y la vida cotidiana, la medicina, la cultura y el ocio, el arte, la música, el cine y el deporte y las tecnologías de la información y la comunicación.

CN.2.10.2. Construye, siguiendo instrucciones precisas, máquinas antiguas y explica su funcionalidad anterior y su prospectiva mediante la presentación pública de sus conclusiones.

c) En 3er. ciclo, tampoco hubo ningún indicador con presencia media o superior. Sin embargo, hubo 3/27 con una "presencia muy baja".

CN.3.5.4. Planifica y realiza experiencias para conocer y explicar las principales características de las reacciones quimicas (combustión oxidación y fermentación) y comunica de forma oral y escrita el proceso y el resultado obtenido.

CN.3.5.3. Construye un circuito eléctrico sencillo aplicando los principios básicos de electricidad y de transmisión de la corriente eléctrica.

CN.3.7.2. Identifica y describe los beneficios y riesgos relacionados con la utilización de la energía: agotamiento, lluvia ácida, radiactividad, exponiendo posibles actuaciones para un desarrollo sostenible.

Solo hubo un indicador con una heterogeneidad destacable:

CN.3.4.1. Pon ejemplos de actuaciones que realizamos los seres humanos diariamente que influyen negativamente sobre el medio ambiente, utilizando adecuadamente los instrumentos para la observación y el análisis de estos actos.

Globalmente podemos decir que el profesorado considera que los libros de texto no contemplan los indicadores del currículum oficial (de hecho, solo uno tiene una presencia media en los tres ciclos). Al ser tan generalizado, no hay un perfil claro de las omisiones. Pero, si nos fijamos en los indicadores con una menor presencia, parece que subyacen conocimientos procedimentales (planificación y realización de experiencias, búsqueda de información, planteamiento de hipótesis...) o actitudinales (adopción de hábitos, valoración de beneficios y riesgos...), lo que, con o sin reforma, resulta preocupante (Pro, 2013b).

Pero, además, se observa que la heterogeneidad de las valoraciones va disminuyendo con el nivel educativo. ¿Es que los libros de las diferentes editoriales se parecen más, a medida que pasan los cursos? Tampoco existe un perfil determinante en relación con la variabilidad, pero, 
como en las omisiones, hay bastantes indicadores en los que subyacen procedimientos y actitudes semejantes a los anteriores.

En cuanto al estudio por editoriales, en la Tabla 4 se recogen los que tienen una mediana menor de 1.5 y una mayor de 2.5 (se ha mantenido la simbología alfanumérica del currículum oficial). Hemos denominado genéricamente EDIT1, EDIT2 y EDIT3 a las tres editoriales con mayor presencia; se ha excluido una porque solo había sido valorada por tres profesores (uno en cada ciclo) y no habría variabilidad. Hemos mantenido la diferenciación por ciclos.

Tabla 4. Opiniones de los docentes sobre los indicadores en las editoriales por ciclo

\begin{tabular}{|c|c|c|c|c|c|c|}
\hline \multirow[b]{2}{*}{ EDITORIAL } & \multicolumn{2}{|c|}{ Primer ciclo } & \multicolumn{2}{|c|}{ Segundo ciclo } & \multicolumn{2}{|c|}{ Tercer ciclo } \\
\hline & Menos de 1.5 & Más de 2.5 & Menos de 1.5 & Más de 2.5 & Menos de 1.5 & Más de 2.5 \\
\hline EDIT1 & & $\begin{array}{l}\text { CN1.2.1, } \\
\text { CN1.4.1, } \\
\text { CN1.5.1, } \\
\text { CN1.5.3, } \\
\text { CN1.6.4, } \\
\text { CN1.7.1, } \\
\text { CN1.7.2, } \\
\text { CN1.8.1 }\end{array}$ & $\begin{array}{l}\text { CN2.1.1, } \\
\text { CN2.4.1, } \\
\text { CN2.5.3, } \\
\text { CN2.9.2 }\end{array}$ & CN2.3.3 & $\begin{array}{l}\text { CN3.3.2, } \\
\mathrm{CN} 3.3 .3, \\
\mathrm{CN} 3.5 .3, \\
\mathrm{CN} 3.5 .4 \\
\mathrm{CN} 3.6 .1 \\
\mathrm{CN} 3.6 .2 \\
\mathrm{CN} 3.7 .1 \\
\mathrm{CN} 3.7 .2 \\
\mathrm{CN} 3.9 .1 \\
\mathrm{CN} 3.9 .2\end{array}$ & $\begin{array}{l}\text { CN3.4.1, } \\
\text { CN3.4.2, } \\
\text { CN3.4.3 }\end{array}$ \\
\hline EDIT2 & $\begin{array}{l}\text { CN1.3.1, } \\
\text { CN1.5.2, } \\
\text { CN1.7.2 }\end{array}$ & & $\begin{array}{l}\text { CN2.1.1, } \\
\text { CN2.2.1, } \\
\text { CN2.2.2, } \\
\text { CN2.2.3, } \\
\text { CN2.2.4, } \\
\text { CN2.3.2, } \\
\text { CN2.4.1, } \\
\text { CN2.4.2, } \\
\text { CN2.6.1, } \\
\text { CN2.6.2, } \\
\text { CN2.8.3, } \\
\text { CN2.9.1, } \\
\text { CN2.9.2, } \\
\text { CN2.10.1 }\end{array}$ & & $\begin{array}{l}\text { CN3.2.1, } \\
\text { CN3.2.3, } \\
\text { CN3.3.1, } \\
\text { CN3.4.2, } \\
\text { CN3.5.4, } \\
\text { CN3.6.1, } \\
\text { CN3.6.2, } \\
\text { CN3.7.1, } \\
\text { CN3.7.2, } \\
\text { CN3.8.2, } \\
\text { CN3.9.2 }\end{array}$ & \\
\hline EDIT3 & $\begin{array}{l}\text { CN1.1.3, } \\
\text { CN1.2.4, } \\
\text { CN1.7.1, } \\
\text { CN1.7.2, } \\
\text { CN1.7.3, } \\
\text { CN1.9.1 }\end{array}$ & $\begin{array}{l}\text { CN1.1.1, } \\
\text { CN1.4.1, } \\
\text { CN1.8.3 }\end{array}$ & $\begin{array}{l}\text { CN2.1.3, } \\
\text { CN2.3.1, } \\
\text { CN2.4.1, } \\
\text { CN2.4.3, } \\
\text { CN2.5.1, } \\
\text { CN2.5.2, } \\
\text { CN2.5.4, } \\
\text { CN2.6.3, } \\
\text { CN2.7.1 }\end{array}$ & & $\begin{array}{l}\text { CN3.2.4, } \\
\text { CN3.4.1, } \\
\text { CN3.4.2, } \\
\text { CN3.7.2 }\end{array}$ & \\
\hline
\end{tabular}

A la vista de los resultados podemos decir:

a) Respecto a la EDIT1, hubo 12/81 indicadores con valores de la mediana que corresponden a una "presencia media", pero 14/81 indicadores - un 17\%- tienen una "muy baja".

b) Respecto a la EDIT2, no hubo NINGÚN indicador con valores iguales o superiores los de una "presencia media"; además, 28/81 indicadores -un 35\%- tienen una "muy baja". 
c) Respecto a la EDIT3, hubo 3/81 indicadores con valores iguales o superiores a los de una "presencia media", pero 19/81 indicadores - casi un 24\%- tienen una "muy baja".

Globalmente podemos decir que 15/243 indicadores -solo un 6\%- tienen una presencialidad aceptable. Parece que EDIT1 es la que se adapta mejor: 11 indicadores con una "presencia media" frente a 9 con una "muy baja". Y la que menos se adapta es EDIT2: solo un indicador tiene una "presencia media", frente a 27 con una "muy baja".

En definitiva, según los participantes, los resultados ponen de manifiesto que los libros -sea cual sea la editorial- no se ajustan a los indicadores establecidos en el currículum oficial.

\section{B) En relación con las competencias}

Otro de los objetivos era conocer la opinión de los participantes sobre si los libros de texto inciden en el desarrollo de competencias. Así pues, hemos analizado la adecuación curricular en cada uno de los ciclos y por editoriales (solo EDIT1, EDIT2 y EDIT3; la otra se excluyó por los motivos apuntados). Nos hemos ocupado de tres que, según el currículum, deberían tener más presencia en el aprendizaje de las Ciencias: CCL, CMyCT y CSyC.

El procedimiento para calcular los valores que representan la presencia de cada competencia viene determinado por la correspondencia que establece el currículum entre los indicadores y las competencias correspondientes. De esta manera, calculamos la presencia media de cada competencia y su desviación típica. En la Tabla 5 se recogen los datos obtenidos.

Tabla 5. Media y desviación típica por competencias, ciclo y editorial

\begin{tabular}{|c|c|c|c|c|c|c|c|}
\hline \multirow[b]{2}{*}{ Competencias } & \multirow[b]{2}{*}{ Editorial } & \multicolumn{3}{|c|}{ Media } & \multicolumn{3}{|c|}{ Desviación típica (dt) } \\
\hline & & $\begin{array}{c}\text { 1ER. } \\
\text { CICLO }\end{array}$ & $\begin{array}{c}2^{\circ} \\
\text { CICLO }\end{array}$ & $\begin{array}{c}\text { 3ER. } \\
\text { CICLO }\end{array}$ & $\begin{array}{c}\text { 1ER. } \\
\text { CICLO }\end{array}$ & $\begin{array}{c}2^{\circ} \\
\text { CICLO }\end{array}$ & $\begin{array}{c}\text { 3ER. } \\
\text { CICLO }\end{array}$ \\
\hline \multirow{3}{*}{ CCL } & EDIT1 & 2,22 & 1,84 & 1,56 & 0,45 & 0,40 & 0,14 \\
\hline & EDIT2 & 2,03 & 1,77 & 1,62 & 0,44 & 0,48 & 0,43 \\
\hline & EDIT3 & 1,98 & 1,77 & 1,79 & 0,42 & 0,36 & 0,43 \\
\hline \multirow{3}{*}{ СMyCT } & EDIT1 & 2,22 & 1,83 & 1,77 & 0,43 & 0,40 & 0,26 \\
\hline & EDIT2 & 1,99 & 1,71 & 1,62 & 0,40 & 0,38 & 0,31 \\
\hline & EDIT3 & 1,93 & 1,80 & 1,69 & 0,27 & 0,39 & 0,27 \\
\hline \multirow{3}{*}{ CSyC } & EDIT1 & 2,22 & 1,94 & 1,94 & 0,46 & 0,44 & 0,37 \\
\hline & EDIT2 & 1,94 & 1,74 & 1,65 & 0,36 & 0,31 & 0,24 \\
\hline & EDIT3 & 1,74 & 1,74 & 1,63 & 0,06 & 0,50 & 0,25 \\
\hline
\end{tabular}

\section{A la vista de los resultados podemos decir:}

a) En 1er. ciclo, la EDIT1 es la que mayor puntuación alcanza en todas las competencias (2.22 en las tres); no se obtienen valores medios iguales o superiores a 2.5. En cuanto a la variabilidad, la EDIT3 parece la más homogénea en sus valoraciones. En cuanto a las competencias, la que tiene mayor presencia es la CCL.

b) En $2^{\circ}$ ciclo, nuevamente EDIT1 tiene mayor presencia en las tres competencias (alcanza entre 1,83 y 1,94); en este caso, con valores medios inferiores a la categoría "Poco". En cuanto a la variabilidad, las tres editoriales presentan valores semejantes. En cuanto a las competencias, la que tiene globalmente mayor presencia es la CSyC. 
c) En 3er. ciclo, la EDIT1 tiene la mayor presencia en dos competencias (CMyCT y CSyC con valores 1,77 y 1,94 respectivamente) y la EDIT3 en la competencia CCL (con 1,79); todos los valores son inferiores a la categoría "Poco". En cuanto a la variabilidad, las valoraciones de EDIT1 son las más homogéneas en los dos primeros ciclos y EDIT2 y EDIT3 en el tercero. En cuanto a las competencias, la que tiene globalmente mayor presencia es nuevamente la CSyC.

Ahora bien, calculado la $\mathrm{T}$ de Wilcoxon entre las tres competencias, no se han detectado diferencias estadísticamente significativas entre ellas (ni globalmente ni por ciclos) para un $\mathrm{p}=0.05$, aunque "a priori" se podría esperar que hubiera una mayor presencia de la CMyCT.

Por otro lado, calculado la U de Mann-Whitney entre los valores de cada competencia de las tres editoriales, en cada uno de los ciclos, podemos decir que no se han encontrado diferencias estadísticamente significativas ( $\mathrm{p}=0,05$ ), salvo 1 de los 27 contrastes realizados (los valores de CSyC de 3er.ciclo de EDIT1 y EDIT2). Nos ha llamado la atención este equilibrio en libros de texto del área de Ciencias de la Naturaleza, aunque lo que desgraciadamente los homogenice sea la escasa presencia de las competencias.

Globalmente, como con los indicadores, parece que NINGUNA editorial contribuye de forma relevante a la adquisición de las tres competencias estudiadas (ninguno alcanza 2,5). No obstante, la editorial que las contempla mejor es la EDIT1, en las tres competencias (en CCL, CMyCT y CSyC), aunque sus diferencias no sean estadísticamente significativas.

\section{Conclusiones e implicaciones}

El objetivo principal de este trabajo era conocer la opinión de unos maestros sobre si los libros de texto responden a los indicadores de los criterios de evaluación y al trabajo por competencias que exige la normativa actual. Para ello, seleccionamos a un grupo de docentes, formados y con experiencia, que valoraron los libros que estaban utilizando. Estos debieron responder un cuestionario construido a partir de los criterios de evaluación y de sus indicadores correspondientes.

A la vista de los resultados obtenidos podemos afirmar que:

- En cuanto a los indicadores de los criterios de evaluación:

- para cada ciclo, los maestros participantes manifiestan que no se ajustan a lo establecido en el currículum oficial; solo un indicador tiene una presencia aceptable;

- dentro de unos valores preocupantes, parece que los libros de 1er. Ciclo contemplan mejor que los de otros ciclos los indicadores de los criterios de evaluación. Igualmente, los libros de la editorial EDIT1 parece que se ajustan algo más que las otras a las exigencias del currículum oficial; $y$,

- podemos decir que no hay un perfil de las omisiones de indicadores, pero parecen subyacer contenidos procedimentales y actitudinales, lo que corrobora los resultados de otras investigaciones (García y Martínez, 2003; Pro Chereguini y Pro Bueno, 2011; Rodríguez, de las Heras, Romero y Cañal, 2014...), realizadas con libros de textos de otras reformas curriculares.

- En cuanto a la contribución a la adquisición de competencias:

- según los maestros de nuestra experiencia, ninguna de las editoriales se ajusta a lo establecido en los programas oficiales; 
- la competencia más presente es: en 1er. ciclo es la CCL y en el $2^{\circ}$ y $3^{\circ}$ está la CSyC. Globalmente todas las competencias aparecen de forma equilibrada, pero llama la atención que la competencia más propia del Área de Ciencias de la Naturaleza -la CMyCT- no destaque en ninguno de los análisis; y,

- en el contexto de valores preocupantes, parece que nuevamente los resultados del 1er Ciclo son más adecuados que los de los restantes.

En otras investigaciones (García-Carmona, Criado y Cañal, 2015) se afirmaba que el estado actual de la educación científica básica en Andalucía no debe limitarse a los materiales didácticos que se diseñan o a la práctica docente en las aulas, sino que debe ocuparse también del programa oficial del que ha de emanar lo anterior. Compartimos, con estos y otros autores, que cada vez se hace más obligada la revisión del currículum actualmente en vigor ya que este presenta errores, carencias, contradicciones y despropósitos que ni se apoyan en experiencias contrastadas ni en marcos teóricos sólidos que lo puedan respaldar.

Pero, además, hay otro motivo: un importante porcentaje del profesorado -que no olvidemos que es el gestor de cualquier reforma-, desconoce qué hay que modificar de lo que se hacía, ignora qué se debe conservar, no sabe qué problemas trata de resolver, etc. ¿Cómo es interpretada la reforma por el profesorado? ¿comparten los profesionales el significado que le atribuyen los legisladores? ¿qué repercusiones tienen estos "cambios" en el estilo de los docentes, en la práctica educativa o en el aprendizaje de los estudiantes?... De hecho, según los resultados de nuestro trabajo, ni siquiera las editoriales han sabido "traducir" los elementos del nuevo paradigma a un recurso tan asentado como los libros de texto.

Hemos de señalar que, pese a las turbulencias que rodean siempre al libro de texto en el ámbito escolar, estos van pasando reforma tras reforma con el único planteamiento didáctico de informar y aplicar los conocimientos en actividades descontextualizadas de la vida del niño. Es cierto que al profesorado le ofrecen orden, control y seguridad ante los cambios legislativos y ante las necesidades de actualización en la materia que debe enseñar. Pero, en la última modificación legislativa, han aparecido términos (competencia, estándar de aprendizaje, indicador...) que apenas ha cambiado la estructura de los libros (Martínez Bonafé y Rodríguez, 2010), con lo que probablemente no cumplirán con la utilidad de orientar a los docentes.

Si echamos un vistazo a los libros de texto de Ciencias de la Naturaleza en Educación Primaria, seguimos observando que, en el fondo, el planteamiento que realizan recuerda a los viejos manuales, como si apenas hayan evolucionado a lo largo de la historia y llevaran al docente a modelos didácticos de enseñanza "tradicional". ¿Es que no se pueden realizar libros de texto que puedan favorecer la innovación, la proximidad a los problemas cotidianos, la cooperación, el interés por lo que se trabaja en el aula, la utilidad de los conocimientos fuera de ella... y que sean coherentes con las tendencias actuales de la Didáctica de las Ciencias Experimentales?

Para algunos autores (López-Martínez y Navarro, 2010), los procedimientos metodológicos utilizados hasta ahora en nuestras aulas -y los libros forman parte de ellos- han procurado uniformizar al educando, homogeneizar a los desiguales... y, a la postre, frenar el desarrollo de las potencialidades del alumno. Parece necesario cambiar la metodología educativa ya que parece que se han modificado los objetivos de aprendizajes (deben simultanearse el saber, el saber hacer, el saber hacer con otros, el saber cuándo y cómo hacer...). Si las editoriales no han sido capaces de plasmar las modificaciones curriculares impuestas desde la Administración, o éstas necesitan una mayor clarificación o precisan de otros planteamientos, estrategias y materiales que actualmente no les proporcionan los actuales libros de texto. La 
situación exige elementos novedosos, originales, el inconformismo con lo disponible, la genialidad, el descubrimiento...; en definitiva, apostar por la innovación y, por supuesto, por la investigación sobre lo que hacemos.

\section{Referencias bibliográficas}

ANELE (2013). Evolución de los precios de los libros de texto.

Bolívar, A. (2015). Un currículum común consensuado en torno al Marco Europeo de Competencias Clave. Un análisis comparativo con el caso francés. Avances en supervisión educativa, (23).

Cano, A. (2015). Una mirada psicológica al enfoque por competencias en educación. Revista Electrónica de Investigación Educativa, 17(2) 1-4. Recuperado de: http://redie.uabc.mx/vol17no2/contenido-canor.html.

Cañal, P., Rodríguez, F. D., Rodríguez, R., y Heras, M. (2014). El conocimiento escolar sobre los animales y las plantas en primaria: Un análisis del contenido específico en los libros de texto. Revista Electrónica de Enseñanza de las Ciencias, 13 (1), 98-115.

Constable, H., Campbell, B., \& Brown, R. (1988). Sectional drawings from science textbooks: an experimental investigation into pupils' understanding. British Journal of Educational Psychology, 58, 89-102.

Cortés, A. L. (2006). Análisis de los contenidos sobre "permeabilidad" en los libros de texto de Educación Primaria. Revista Electrónica de Enseñanza de las Ciencias, 5(1), 136-160.

Eurydice (2011). Science Education in Europe: National Policies, Practices and Research. http://eacea. ec.europa.eu/education/eurydice.

Ferreyra, H. y Millen, D. (2006). Competencias educativas prioritarias. Novedades Educativas. 180, 33-35.

Ferreyra, H., Orego, S. A., Peretti, G. C., Fontana, M., Pasut, M., Seara, R. C. C., Caelles, S., Eberle, M. J., Kowadlo, M. J., Bodoira, S. M., Millen, D. M. y Schneider, C. S. (2008). De aprendizajes, competencias y capacidades en la educación primaria. Desandando caminos para construir nuevos senderos. Revista Iberoamericana de Educación, 3(47), 1-13. Recuperado de: http://rieoei.org/2702.htm.

García, S. y Martínez, C. (2003). Análisis del trabajo práctico en textos escolares de primaria y secundaria. Enseñanza de las Ciencias, número extra 2003, 5-16.

García, A., Criado, A. M. y Cañal, P. (2015). La educación científica en el currículo oficial de Primaria para Andalucía: Un análisis crítico. Revista Investigación en el Escuela, 87, 5-19.

Gliem, J. A., \& Gliem, R. R. (2003). Calculating, interpreting, and reporting Cronbach's alpha reliability coefficient for Likert-type scales. Midwest Research to Practice Conference in Adult, Continuing, and Community Education.

Ley Orgánica 8/2013, de 9 de diciembre, para la mejora de la calidad educativa -LOMCE-. (BOE del 10-12-2013, Num. 295, pp. 97858 a 97921).

López, A., y Postigo, Y. (2014). Análisis de las imágenes del cuerpo humano en libros de texto españoles de primaria. Enseñanza de las ciencias: revista de investigación y experiencias didácticas, 32(3), 551-570. 
López, A. y Postigo, Y. (2016). ¿Qué libro de texto elegir? La competencia visual en las actividades con imágenes. Revista Eureka sobre Enseñanza y Divulgación de las Ciencias, 13 (1), 84-101. doi: 10.25267/Rev_Eureka_ensen_divulg_cienc.2016.v13.i1.07.

López Martínez, O., y Navarro Lozano, J. (2010). Creatividad e inteligencia: un estudio en Educación Primaria. Revista de Investigación Educativa, 28(2), 283-296.

Martin, C., Jiménez, M. y Prieto, T. (2015). Tendencias del profesorado de ciencias en formación inicial sobre las estrategias metodológicas en la enseñanza de las ciencias. Estudio de un caso en Málaga. Enseñanza de las Ciencias, 33(1), 167-184.

Martínez Bonafé, J. (2002). Políticas del libro de texto escolar. Madrid: Morata.

Martínez, B. y Gil, M. J. (2001). The importance of images in astronomy education. International Journal of Science Education, 23(11), 1125-1135.

Martínez, J. y Rodríguez, J. (2010). Fundamentos de una psicología El currículum y el libro de texto. Una dialéctica siempre abierta. En Gimeno, J. (Comp.), Saberes e incertidumbres sobre el curriculum (pp.246-296). Madrid: Morata.

MC Millan, J. y Schumacher, S. (2005). Investigación educativa. Madrid: Pearson Education.

Méndez, D., Méndez, A. y Fernández, F. J. (2015). Análisis y valoración del proceso de incorporación de las Competencias Básicas en Educación Primaria. Revista de Investigación Educativa, 33(1), 233-246.

Molina Jaén, M. D., Rodríguez Moreno, J., Pérez Navío E., y Callado Moreno, J. A. (2015). Incidencia de la formación en el profesorado. Webquest: una propuesta metodológica en educación infantil y primaria. Edutec. Revista Electrónica de Tecnología Educativa, 52, 1 17. Recuperado de: http://www.edutec.es/revista/index.php/edutece/article/viewFile/601/pdf_35.

Molina Jaén M. D. y Rodríguez Moreno, J. (2016). El desarrollo de las competencias en los libros de texto de educación infantil. Cuestionario de valoración. Dedica. Revista de Educação e Humanidades, (10), 199-213.

Monereo, C. y Pozo, J. I. (2007). Competencias básicas. Cuadernos de pedagogía, 370, 10-18.

Orden de 17 de marzo de 2015, por la que se desarrolla el currículo correspondiente a la Educación Primaria en Andalucía (BOJA del 27-3-2015, Num. 60, pp. 9-72).

Orden ECD/65/2015, de 21 de enero, por la que se describen las relaciones entre las competencias, los contenidos y los criterios de evaluación de la educación primaria, la educación secundaria obligatoria y el bachillerato (BOE del 29-1-2015, Num.25, pp. 6986-7003).

Perales, F.J. (2006). Pasado, presente y ¿futuro? de los libros de texto. Alambique, 48, 57-63.

Perrenoud, Ph. (2004). Diez nuevas competencias para enseñar. Barcelona. Graó.

Pro Bueno, A. (2013a). LOMCE y física contemporánea. Alambique, 75, 5-8.

Pro Bueno, A. (2013b). Enseñar procedimientos: por qué y para qué. Alambique, 73, 69-76.

Pro Bueno, A., Sánchez Blanco, G. y Valcárcel Pérez, M.V. (2008). Análisis de los libros de texto de física y química en el contexto de la reforma LOGSE. Enseñanza de las ciencias, 26(2), 193-209.

Pro Bueno, A. y Miralles, P. (2009). El currículum de Conocimiento del Medio Natural, Social y Cultural en la Educación Primaria. Educatio Siglo XXI, 27(1), 59-96. 
Pro Bueno, A. y Rodríguez Moreno, J. (2011) Realizando experimentos para aprender en educación primaria. Aula de innovación educativa, 207, 28-34.

Pro Bueno, A. y Rodríguez Moreno, J. (2014). Ahorrando energía en Educación Primaria: estudio de una propuesta de enseñanza. Enseñanza de las ciencias, 32(2), 151-170.

Pro Chereguini, C. y Pro Bueno, A. (2011). ¿Qué estamos enseñando con los libros de texto? La electricidad y la electrónica de Tecnología en $3^{\text {a }}$ ESO. Revista Eureka sobre Enseñanza y Divulgación de las Ciencias, 8(2). doi: 10.25267/Rev_Eureka_ensen_divulg_cienc.2011.v8.i2.02

Pro Chereguini, C., Pro Bueno, A. y Serrano Pastor, F. (2016). ¿Cómo utilizan sus conocimientos científicos sobre "Dispositivos y máquinas mecánicas" los futuros maestros cuando realizan una actividad de laboratorio? Revista Electrónica Interuniversitaria de Formación del Profesorado, 19(3), 77-89. doi: 10.6018/reifop.19.3.267261

Qadeer, A. (2014). Un estudio de libros de texto de electricidad de $6^{\circ}$ grado: respuestas de los estudiantes y análisis de contenido. Enseñanza de las ciencias: revista de investigación y experiencias didácticas, 32(1), 285-286.

Rodríguez Lorenzo, M. Á. (2015). "Abandoné las aulas de Historia sin ver ni un mapa, ni una lámina, ni un libro que no fuera el de texto" (Estudiar historia en la España del siglo XIX). Presente y Pasado. Revista de Historia, 20(39), 103-120. Recuperado de: https://www.saber.ula.ve/bitstream/123456789/40933/1/articulo4.pdf.

Rodríguez, F., de las Heras, M. Á., Romero, R., y Cañal, P. (2014). El conocimiento escolar sobre los animales y las plantas en primaria: Un análisis del contenido específico en los libros de texto. Revista Electrónica de Enseñanza de las Ciencias,13(1), 97-114. Recuperado de: http://reec.uvigo.es/volumenes/volumen13/REEC_13_1_6_ex761.pdf.

Rodríguez, A., Ruiz, S. y Guerra, Y. (2007). Competencias ciudadanas aplicadas a la educación. Revista Educación y Desarrollo Social, 1(1), 141-157. Recuperado de: http://www.unimilitar.edu.co/documents/63968/80132/RevNo1vol1.Art10.pdf

Shayer, M. y Adey, P. (1984). La ciencia de enseñar ciencias. Madrid: Narcea.

Strube, P. (1989). The notion of style in physics textbooks. Journal of Research in Science Teaching, 26, 777-779.

Travé, G., Estepa, J. y Delval, J. (2015). Análisis de la fundamentación didáctica de los libros de texto de Conocimiento del medio social y cultural. Educación XXI. doi:10.5944/educxx1.11831

Trujillo, F. (2014). La LOMCE analizada. Recuperado de http://www.stecyl.es/borralex/LOMCE/analisis_LOMCE_@ftsaez.pdf.

Urones, C., Escobar, B., y Vacas, J. M. (2013). Las plantas en los libros de Conocimiento del Medio de 2o ciclo de primaria. Revista Eureka sobre Enseñanza y Divulgación de las Ciencias, 10(3), 328-352. doi: 10.25267/Rev_Eureka_ensen_divulg_cienc.2013.v10.i3.03

Valera, M. (2010). Sobre los manuales escolares. Escuela Abierta, 13, 97-114.

Villarini, A. R. (2000). El currículum del desarrollo bumano: currículum básico de español. San Juan PR: Biblioteca del Pensamiento Crítico. 


\begin{abstract}
\begin{tabular}{|l|l|llll}
\hline ÁREA: CIENCIAS DE LA NATURALEZA & CICLO: $1^{\circ}$ & EDITORIAL: & ANAYA SANTILLANA SM EDELVIVES \\
\hline
\end{tabular}
C.E.1.1. Obtener información y realizar pequeñas conjeturas sobre hechos y elementos naturales previamente delimitados $y$ realizar sencillos experimentos que faciliten su comprensión, potenciando el trabajo cooperativo y expresando oralmente
\end{abstract} los resultados obtenidos.

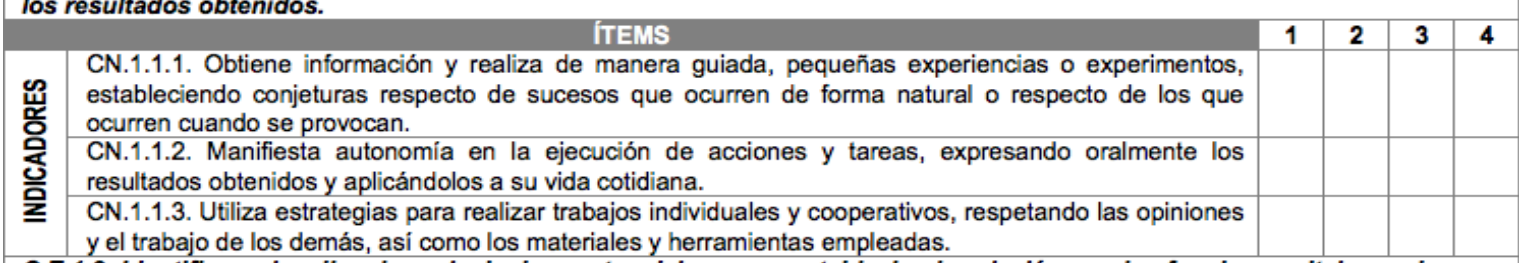
C.E.1.2. Identificar y localizar las principales partes del cuerpo, estableciendo relación con las funciones vitales en las que se ven implicadas, para potenciar hábitos saludables básicos poniendo ejemplos asociados a la higiene, la alimentación equilibrada, el ejercicio físico y el descanso como formas de mantener la salud, el bienestar y el buen funcionamiento del cuerpo.

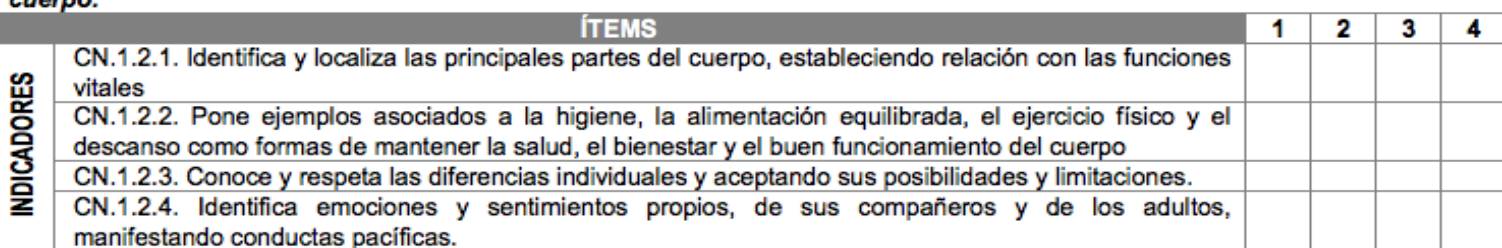

C.E.1.3. Identificar y clasificar los seres vivos del entorno en animales y plantas, conociendo su estructura y señalando la importancia del agua para la vida, desarrollando valores de cuidado y respeto.

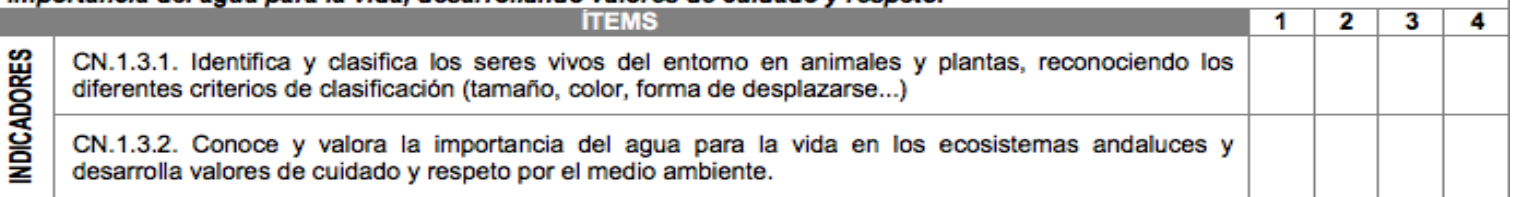

C.E.1.4. Observar los diferentes seres vivos del entorno más cercano, utilizando diferentes instrumentos que permitan

despertar comportamientos de defensa, respeto y cuidado hacia los seres vivos.

in CN.1.4.1. Conoce y utiliza de forma adecuada diferentes instrumentos para la observación y el estudio de los seres vivos.

CN.1.4.2. Manifiesta en su vida cotidiana comportamientos de defensa, respeto y cuidado hacia los seres vivos de su entorno.

C.E.1.5. Observar, identificar, diferenciar y clasificar materiales de su entorno según propiedades físicas elementales relacionándolas con su uso. Reconocer efectos visibles de las fuerzas sobre los objetos.

$$
\text { IIEMS }
$$

ü estado, origen, olor, sabor, textura, color, etc.

materiales por sus propiedades elementales: forma,

CN.1.5.2. Relaciona algunas de las propiedades elementales de los materiales con sus usos.

CN.1.5.3. Observa y predice el resultado de la aplicación de fuerzas sobre objetos respecto a la dirección de su movimiento.

C.E.1.6. Conocer las propiedades elementales del magnetismo y las principales leyes que rigen el cambio de estado de la materia, mediante la realización, de forma guiada y colaborativa, de investigaciones y experiencias sencillas a través del método científico, así como comunicar oral y gráficamente las conclusiones obtenidas.

$$
\text { ITEMS }
$$

CN.1.6.1. Observa e identifica las principales características de los imanes

i CN.1.6.2. Aplica el método científico en su trabajo, es capaz de preguntar y formula hipótesis y realiza

щّ experiencias para elaborar conclusiones sobre las propiedades del imán y los principios del 응 magnetismo.

S CN.1.6.3 Observa, identifica y describe oralmente y por escrito los cambios de estado del agua.

든 CN.1.6.4. Realiza sencillas experiencias y elabora textos, presentaciones y comunicaciones como técnica para el registro de un plan de trabajo, comunicando de forma oral, escrita y audiovisual las conclusiones.

C.E.1.7. Realizar de forma individual y en grupo experiencias sencillas de reutilización y reciclado de materiales para tomar

\begin{tabular}{|c|c|c|c|c|c|}
\hline \multicolumn{2}{|r|}{ ITEMS } & 1 & 2 & 3 & 4 \\
\hline \multirow{3}{*}{ 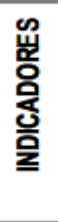 } & $\begin{array}{l}\text { CN.1.7.1. Observa e identifica las prácticas que producen residuos, contaminan y producen impacto } \\
\text { ambiental. }\end{array}$ & & & & \\
\hline & $\begin{array}{l}\text { CN.1.7.2. Identifica, valora y muestra conductas responsables de ahorro, reutilización y reciclaje de } \\
\text { materiales en el colegio, en casa y en el entorno. }\end{array}$ & & & & \\
\hline & $\begin{array}{l}\text { CN.1.7.3. Realiza sencillas experiencias y elabora textos, presentaciones y comunicaciones como } \\
\text { técnica para el registro de un plan de trabajo, comunicando de forma oral, escrita y audiovisual las } \\
\text { conclusiones. }\end{array}$ & & & & \\
\hline
\end{tabular}
conciencia del uso adecuado de los recursos. 


\begin{tabular}{|c|c|c|c|c|c|}
\hline \multicolumn{2}{|r|}{ ITEMS } & 1 & 2 & 3 & 4 \\
\hline \multirow{4}{*}{$\begin{array}{l}\text { 吕 } \\
\text { 옹 } \\
\text { 웛 } \\
\text { 을 }\end{array}$} & CN.1.8.1 Observa, identifica y describe algunos componentes de máquinas y aparatos de su entorno. & & & & \\
\hline & $\begin{array}{l}\text { CN.1.8.2. Observa e identifica alguna de las aplicaciones de las máquinas y aparatos, y su utilidad para } \\
\text { facilitar las actividades humanas. }\end{array}$ & & & & \\
\hline & $\begin{array}{l}\text { CN.1.8.3. Valora y describe la influencia del desarrollo tecnológico en las condiciones de vida y en el } \\
\text { trabajo. }\end{array}$ & & & & \\
\hline & $\begin{array}{l}\text { CN.1.8.4. Identifica los elementos básicos de un ordenador y se inicia de forma guiada en el uso de } \\
\text { internet. }\end{array}$ & & & & \\
\hline \multirow{3}{*}{$\begin{array}{l}\text { 吕 } \\
\text { 음 } \\
\text { 옴 } \\
\text { 을 }\end{array}$} & ITEMS & 1 & 2 & 3 & 4 \\
\hline & $\begin{array}{l}\text { CN.1.9.1. Monta y desmonta algunos objetos y aparatos simples, describiendo su funcionamiento, } \\
\text { piezas, secuencia de montaje y explicando su utilización de forma segura. }\end{array}$ & & & & \\
\hline & $\begin{array}{l}\text { CN.1.9.2. Mantiene conductas seguras tanto en el uso como en el montaje y desmontaje de objetos } \\
\text { simples. }\end{array}$ & & & & \\
\hline
\end{tabular}

